\title{
AstroSat/LAXPC view of GX 17+2: Spectral Evolution along the Z-track
}

\author{
V. K. Agrawal ${ }^{1}$ \\ vivekag@ursc.gov. in \\ Anuj Nandi ${ }^{1}$ \\ anuj@ursc.gov.in \\ and \\ M. C. Ramadevi ${ }^{1}$ \\ ramadevi@ursc.gov.in
}

\begin{abstract}
In this paper, we present the first results obtained using $\sim 50$ ks observations of the bright low-mass X-ray binary (LMXB) GX 17+2 with Large Area X-ray Proportional Counter (LAXPC) onboard AstroSat. The source traced out a complete Z-track in the hardness intensity diagram (HID). The spectra at different sections of the Z-diagram are well described by either a combination of a thermal Comptonization component, a power-law and a relativistic iron line or a model consisting of a thermal disk component, a single temperature blackbody, a powerlaw and a relativistic iron line. Fitting the spectra with both phenomenological models suggests that the power-law component is strong in the horizontal branch (HB), becomes weaker as the source moves down the normal branch (NB) and then again becomes stronger as the sources moves up the flaring branch (FB). However, we find that the strength of the power-law component is model dependent, although the trend in the variation of the power-law strength along the Z-track is similar. A simple model composed by a Comptonized emission and power-law component, convolved with the ionized reflection, also describes the spectra very well.

A normal branch oscillation (NBO) with a centroid frequency $7.42 \pm 0.23 \mathrm{~Hz}$, quality factor $(\mathrm{Q}) \sim 4.88$, rms $1.41 \pm 0.29 \%$ and significance $5.1 \sigma$ is detected
\end{abstract}

\footnotetext{
${ }^{1}$ Space Astronomy Group, ISITE Campus, U. R. Rao Satellite Center, Bangalore, 560037, India
} 
at the middle of the NB. The parameters of the Comptonized emission show a systematic evolution along the Z-diagram. The optical depth of the corona increases as the source moves up along the FB, suggesting possible trigger of an outflow or dumping of the disc material in to the corona by radiation pressure.

Subject headings: accretion, accretion discs - X-rays: binaries - X-rays: individual: GX $17+2$

\section{Introduction}

Z-sources, a subclass of neutron star low-mass X-ray binaries (NS-LMXBs), are among the most luminous and persistent X-ray sources in the sky. They have X-ray luminosities typically in the range of $0.5-1.0$ times the Eddington limit $\left(L_{E d d}\right)$ for $1.4 M_{\odot}$ neutron star. They are named so as they trace out a ' Z' pattern (Hasinger and van der Klis 1989) in the color-color diagram (CCD) and the hardness intensity diagram (HID). From the top left, three branches of the Z-track are: horizontal branch (HB), normal branch (NB) and flaring branch (FB). Another subclass of NS-LMXBs is atoll sources. They trace out a 'C-type' pattern in the CCD and the HID. The transient Z-source XTE J1701-462 (Remillard et al. 2006) showed both atoll and Z-source like behaviour at different flux levels, suggesting that both are similar system accreting at different rates (Homan et al. 2007; Lin, Remillard and Homan 2009).

Though spectral properties of these sources have been investigated in detail, results of spectral modelling are not conclusive and model degeneracy exists. Between two widely accepted scenario, one evokes a model which is sum of a multicolour blackbody emission from a standard disc and a component resulting from inverse Compton scattering of the soft seed photons by hot plasma in the boundary-layer/central-corona (Mitsuda et al. 1984; Barret 2001; Di Salvo et al. 2002; Agrawal and Sreekumar 2003; Agrawal and Misra 2009). In another scenario, the emitted spectrum is modeled as sum of a single temperature blackbody component from the boundary-layer/NS-surface and the hard Comptonized component from the hot inner accretion flow (Di Salvo et al. 2000, 2001; Sleator et al. 2016). The choice of the soft component between the multicolour disc emission (first scenario) and the blackbody emission from the neutron star surface (second scenario) makes these two approaches different. Though, the Comptonized component in both approaches is described by same model, it may be associated with different parts of the accretion flow. Recent work by Lin et al. (2012) has shown that sum of a multi-colour disc emission and a blackbody emission also provides acceptable description of the X-ray spectra of NS-LMXBs. However, this model is more appropriate for the spectra without hard tails or high energy coverage. A non-thermal 
power-law component has been reported in the energy spectra of five persistent Z-sources (Cyg X-2, Di Salvo et al. 2002; GX 17+2, Di Salvo et al. 2000; Farinelli et al. 2005; Sco X-1, Revnivtsev et al. 2014; Di Salvo et al. 2006; D'Ai et al. 2007; D'Amico et al. 2001; GX 5-1, Asai et al. 1994; GX 349+2, Di Salvo et al. 2001; and the peculiar source Cir X-1, Iaria et al. 2001) and one transient Z-source XTE J1701-462 (Ding et al. 2011). A hint of a power-law component is also present in the BeppoSAX spectrum of the Z-source GX 340+0 (Iaria et al. 2006).

Investigations of the fast time variability revealed rich varieties of Quasi-periodic Oscillations (QPOs) and noise components in the power density spectra (PDS) of the Z-sources (van der Klis 1995). These features are tightly correlated with the positions on the Z-diagram (Jonker et al. 2000, 2002; Homan et al. 2002). QPOs in the frequency range $15-80 \mathrm{~Hz}$ are seen in the HB and the upper part of NB. They are called horizontal branch oscillations (HBOs). QPOs in the frequency range $5-7 \mathrm{~Hz}$ are also seen in the middle part of $\mathrm{NB}$ to the lower part of $\mathrm{NB}$ and are called normal branch oscillations (NBOs). The frequency of NBO suddenly increases to $15-30 \mathrm{~Hz}$ as the source crosses the NB/FB vertex. It is believed that at the NB/FB vertex $\mathrm{NBO}$ is transformed into flaring branch oscillations (FBOs). A pair of $\mathrm{kHz}$ QPOs in the frequency range $200-1200 \mathrm{~Hz}$ have been seen in the six persistent Z-sources (Sco X-1, van der Klis et al. 1996; Cyg X-2, Wijnands et al. 1998a; GX 5-1, Wijnands et al. 1998b; GX 340+0, Jonker et al. 1998; GX 17+2, Wijnands et al. 1997; GX 349+2, Zhang, Strohmayer and Swank 1998) and the transient Z-source (Sanna et al. 2010). The noise components observed in the Z-sources are: Very low frequency noise (VLFN), low frequency noise (LFN) and high frequency noise (HFN). LFN and HFN are band limited noise and are modeled with a cutoff power-law or a Lorentzian component and VLFN is modeled with a power-law. A smooth evolution of the power spectral features along the CCD suggests that the energy spectra should also vary smoothly along the Z-track. Studies have been carried out to understand the evolution of the X-ray spectral parameters along the Z-track (Di Salvo et al. 2000, 2002; Done. Zvcki and Smith 2002; Agrawal and Sreekumar 2003; Agrawal and Misra 2009; Lin et al. 2012). There are also attempts to investigate the correlation between the spectral parameters and the parameters of the power spectral features (Titarchuk, Kuznetsov \& Shaposhnikov 2007; Lin et al. 2012).

GX $17+2$ is a bright and persistent NS-LMXB classified as Z-source (Hasinger and van der Klis 1989). The source has exhibited all types of QPOs (HBO, NBO, FBO and pair of $\mathrm{kHz}$ QPOs) so far identified in the Z-sources. A detailed temporal evolution along the Z-track has been investigated using the Rossi X-ray Timing Explorer (RXTE) data (Homan et al. 2002). A detailed X-ray spectral studies have been carried out previously using the $B e p$ poSAX (Di Salvo et al. 2000) and RXTE data (Lin et al. 2012). Lin et al. (2012) fitted the X-ray spectra with a model consisting of a single temperature blackbody (bbodyrad in 
XSPEC), a multi-colour disc component (diskbb in XSPEC), a weak Comptonized component described by cutoff powerlaw and a Gaussian line with energy fixed at $6.6 \mathrm{keV}$. They noted that frequency of the upper $\mathrm{kHz}$ QPO was correlated with the inner disc radius. BeppoSAX spectra of the source were fitted with a blackbody and Comptonized component (Di Salvo et al. 2000). A power-law tail with photon index $\sim 2.7$ was also found to be present in the HB spectra of the source, perhaps correlated with the radio (jet) emission (see, Migliari et al. 2007). Further, a signature of reflection feature was also seen during the NuSTAR observations of this source (Ludham et al. 2017). Modelling of the NuSTAR spectrum with the reflection model suggested a low inclination $\left(25-38^{\circ}\right)$ of the system. An inclination angle of $\sim 30^{\circ}$ for this neutron star system was derived by fitting the relativistic iron line observed with the Suzaku data (Cackett et al. 2010). Analysis of the NuSTAR observation (Ludham et al. 2017) also suggested that the accretion disc is truncated close to the inner most stable circular orbit (ISCO).

In this paper, we report the results obtained by the first AstroSat/LAXPC observations of the source GX $17+2$. We present the spectral and temporal evolution of the source along the Z-track utilizing broad band $(3-80 \mathrm{keV})$ data from the LAXPC instrument. The source traced a Z-track in the HID during the observations and showed the presence of a QPO in the normal branch. We report the detection of a hard power-law tail in both $\mathrm{HB}$ and FB branch of the Z-track. The paper is organized as follows. The observations and data reduction are described in $\S 2$. $\S 3$ deals with the methods of the data analysis and modelling of the temporal and spectral data. The results of spectral and timing analysis are presented in $\S 4$. In section $\S 5$, we interpret our results and conclude.

\section{Observations and Data Reduction}

The source GX 17+2 was observed from May 11, 2016 to May 14, 2016 with Large Area X-ray Proportional Counter (LAXPC) onboard AstroSat. The data was collected during Guaranteed Time (GT) observation. The source was observed for a total exposure time of $50 \mathrm{ks}$. LAXPC instruments provide high time resolution (10 micro second) and moderate energy resolution data in the $3-80 \mathrm{keV}$ band. Three co-aligned identical proportional counters (LAXPC10, LAXPC20 and LAXPC30) provide a combined effective area of $\sim 6000$ $\mathrm{cm}^{2}$ at $15 \mathrm{keV}$ (Yadav et al. 2016; Agrawal et al. 2017; Antia et al. 2017). The detection efficiency $>50$ per cent above $30 \mathrm{keV}$ is achieved by the LAXPC detection volume, which is a mixture of xenon and methane at 2 atmospheric pressure. For the data reduction, we use the software and follow the procedures provided in the webpage http://astrosat-ssc.iucaa.in/. We use recent calibration files and background models provided by the instrument team (see 
Antia et al. (2017) for more details).

\section{Data Analysis}

\subsection{Lightcurve and Z-track}

The background subtracted binned lightcurve in the energy range $3.0-60.0 \mathrm{keV}$ is created using the LAXPC10 event mode data. The average background count rate varies between $170-190$ counts/s in the energy band of $3.0-60.0 \mathrm{keV}$. We use a bin size of 256 sec for the lightcurve generation. An investigation of the lightcurve reveals that initially the source intensity is roughly constant $(1900-2000 \mathrm{cts} / \mathrm{s})$ and then the source intensity

gradually decreases from $\sim 1900 \mathrm{cts} / \mathrm{s}$ to $\sim 1500 \mathrm{cts} / \mathrm{s}$. This decaying phase is interrupted by a sudden increase (flare) in the source count rates (see Fig. 1).

Hardness Intensity Diagram (HID) is created using the background subtracted lightcurves in the $3.0-20.0 \mathrm{keV}, 7.3-10.5 \mathrm{keV}$ and $10.5-20.0 \mathrm{keV}$ energy bands. Here, the hard colour is defined as the ratio of count rates in the energy bands $10.5-20.0 \mathrm{keV}$ and $7.3-10.5 \mathrm{keV}$. The energy bands used to define the hard colour and intensity are similar to that used by Homan et al. (2002) for a comparison. To create the HID, we plot the hard-colour against the source intensity in the energy band $3.0-20.0 \mathrm{keV}$. The HID is shown in Figure 2. All the three branches of the Z-track can be identified clearly in the HID. To study the spectral and temporal evolution along the Z-track, we divide the Z-track into 9 segments. The horizontal branch (HB) is divided into two parts 'HB1' and 'HB2'. The normal branch (NB) is divided into three parts 'NB1', 'NB2' and 'NB3'. The flaring branch (FB) is divided into total 4 divisions, 'FB1', 'FB2', 'FB3' and 'FB4'. These sections are marked in the HID. The top left part of the HB (HB1) is almost horizontal. However, the HB2 section is almost vertical and it continues to bend left into the NB. For each of these sections, we create the X-ray spectra and PDS (Power Density Spectra). These spectra and PDS are used for further spectral and temporal analysis.

\subsection{Spectral Analysis}

First, we create the source and background spectra for the different sections of the HID. The background subtracted spectra are fitted using the spectral fitting software XSPEC version 12.9.1. Since LAXPC10 is well calibrated and have less background issues, we use data from this detector only to perform the spectral fitting. Unless mentioned explicitly, all the errors are computed using $\Delta \chi^{2}=1.0$ (68\% confidence level). We included $1 \%$ systematics 


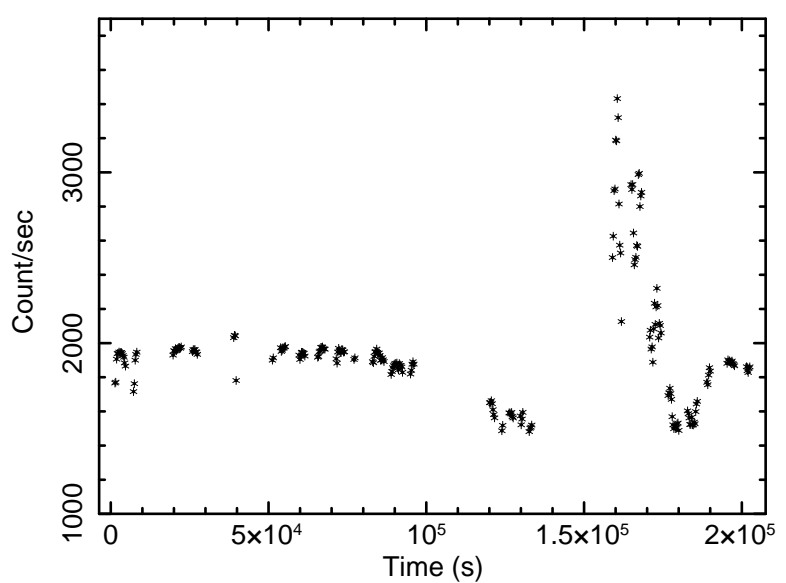

Fig. 1.- Background subtracted lightcurve of GX $17+2$ in the energy band $3.0-60.0 \mathrm{keV}$. The lightcurve is created using $256 \mathrm{sec}$ binsize. Only LAXPC10 has been considered for creating the lightcurve. The errors on the data points are smaller than the marker size.

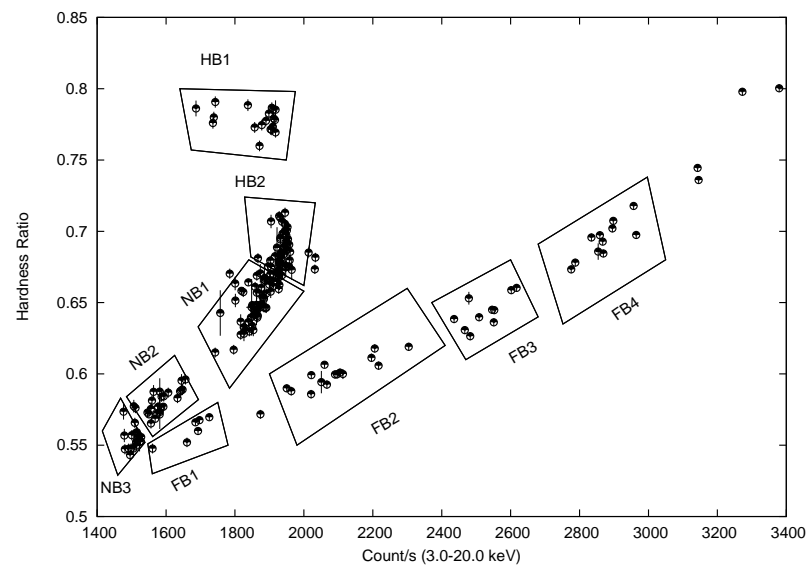

Fig. 2.- Hardness Intensity Diagram (HID) of GX 17+2 created using LAXPC10 data. Each point on the HID corresponds to $256 \mathrm{sec}$ binsize. The marked regions show the sections used to create the energy spectra and power density spectra. See text for details. 
in overall fitting to account for the uncertainty in the spectral response (Agrawal et al. 2018).

We attempt multi-component phenomenological spectral models to fit the X-ray spectra of the source in HB1. First, we fit the data with the combination of a thermal Comptonization and a broad Gaussian line. To represent the Comptonized emission, we use nthComp model of XSPEC (Zdziarski et al. 1996). In our model, we assume the source of the seed photon as blackbody emission. The fitting gives the reduced $\chi^{2}\left(\chi^{2} /\right.$ dof $)=109 / 81$. We find that there is an excess of emission above $30 \mathrm{keV}$ on the top of the nthComp model. To account for this excess emission, we include a power-law component which improved the fit significantly $\left(\chi^{2} / d o f=66 / 79\right)$ with a chance improvement probability of $2.5 \mathrm{e}-9$. The parameters of the Gaussian component and reduced $\chi^{2}$ obtained by fitting the data with wabs (nthcomp +Gauss+power-law) model are given in Table 2.

We also model the broad Gaussian feature with diskline model (Fabian et al. 1989) which takes into account the relativistic effect for a Schwarzschild space-time geometry. The diskline model has 6 free parameters: inner disc radius, outer disc radius, line energy, inclination, emissivity index and normalization. We kept the inner disc radius, normalization and line energy free. The inclination angle derived from the NuSTAR data of this source varies between $\sim 25-40^{\circ}$ (Ludham et al. 2017) and hence we allow to vary the inclination angle between these values. We find that inclination angle peges at $40^{\circ}$. Hence, we fix the inclination at this value. We also fix the emissivity index at -3 and outer disc radius at 1000 gravitational radii. Fitting the iron line feature with diskline model, we get a similar reduced $\chi^{2}\left(\chi^{2} / d o f=67 / 79\right)$ with respect to the fit with a Gaussian profile and hence we adopt wabs (nthComp + diskline+power-law) as the best description of the GX 17+2 spectrum at the section HB1. We use this model to fit the spectra from other sections of Z-track and find it adequate (see Table 1). We refer to this model as Model 1.

We also tried a multi-component model, consisting of emission from a multi-colour disk, a blackbody emission component from boundary-layer/NS-surface and a relativistic iron line to fit the spectra at different sections of the Z-track. A significant power-law component is also required in order to fit the spectra at different parts of the Z-track (see Table 3). To model the emission from disc, we use diskbb model of XSPEC and to represent the blackbody we use bbodyrad model in XSPEC. We find that diskbb+bbodyrad+power-law+diskline model also successfully describes the spectra of this source. We refer to this model as Model 2. To understand the nature of iron line we also tried a convolution model rfxconv which takes into account the reflection from an ionized disk (Kolehmainen. Done \& Trigo 2011). We refer to the model wabs ${ }^{*} r f x c o n v^{*}(n t h c o m p+p o w e r-l a w)$ as Model 3. We allow the inclination angle to vary between $25^{\circ}$ to $40^{\circ}$ (see Ludham et al. (2017)). The inclination angle peges to the lower limit $25^{\circ}$ and hence we fix the inclination at $25^{\circ}$ while fitting the spectra with this 

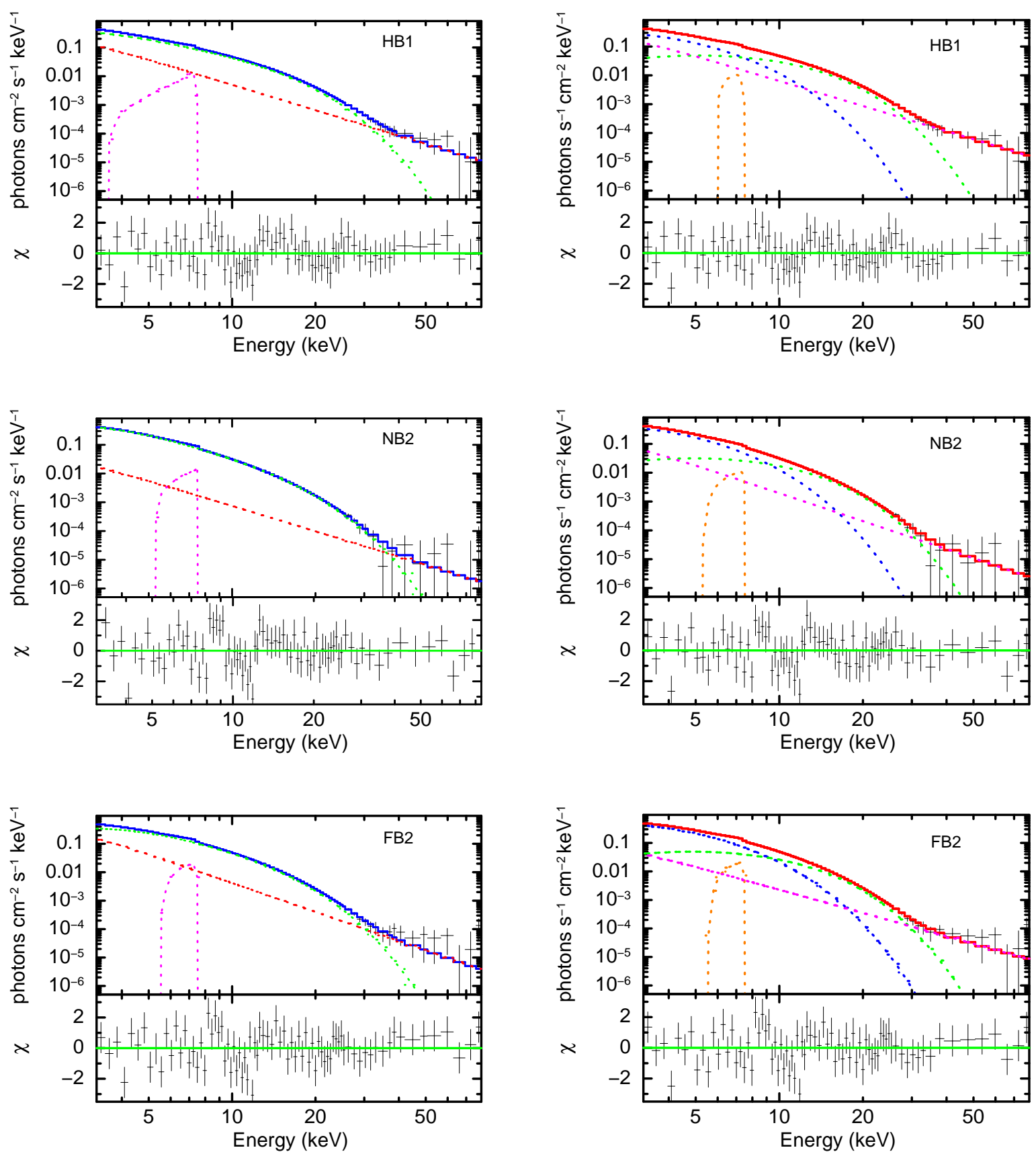

Fig. 3.- The unfolded spectra Model 1: nthComp+diskline+power-law on the left panel and Model 2: diskbb+bbodyrad+diskline+power-law on the right panel of GX 17+2 at three different parts of the Z-track. The top panel shows the spectra at HB1. A clear signature of a hard tail extending above $30 \mathrm{keV}$ is visible in this panel. The bottom panel displays the spectra at FB2 showing the signature of hard tail. The middle panel, which shows the spectra at NB2, suggests that hard tail is weak in the NB. 

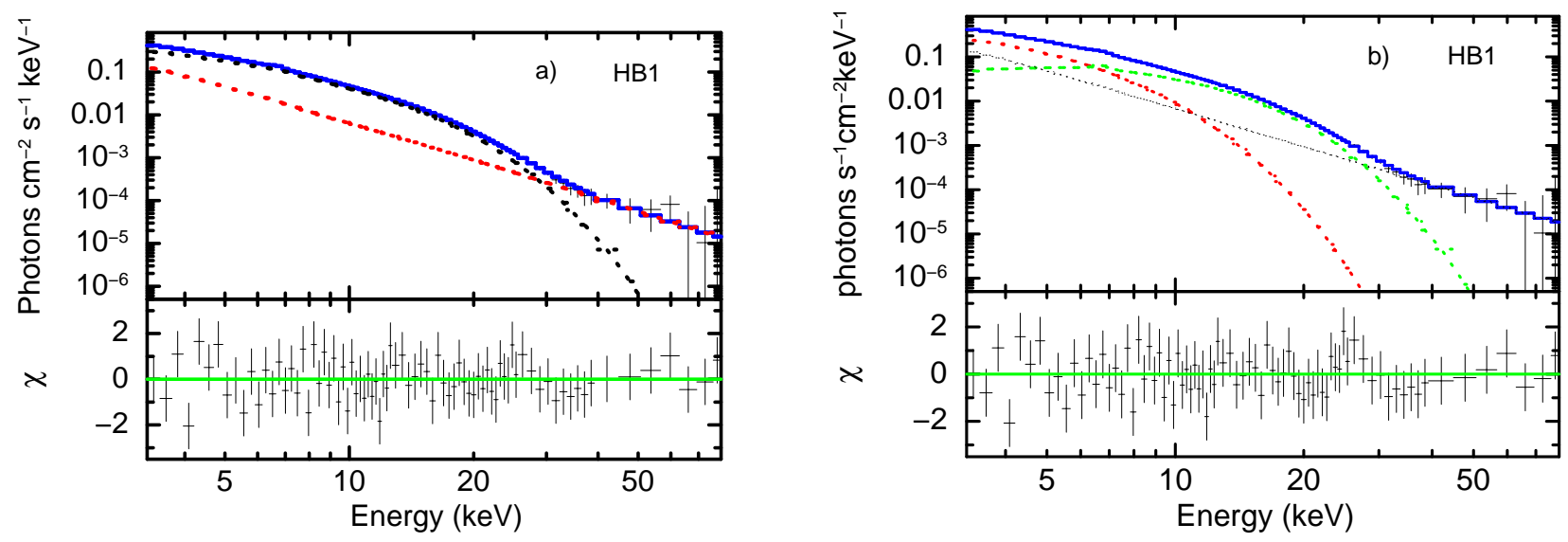

Fig. 4. - a) The unfolded spectrum for HB1 section and the used model is Model 3 : rfxconv* (nthComp+power-law). b) The unfloded spectrum for the HB1 and the used model is Model $4:$ diskbb+power-law+relconv ${ }^{*} b b r e f l$. The residuals in unit of sigma are shown in the bottom panels of a) and b).
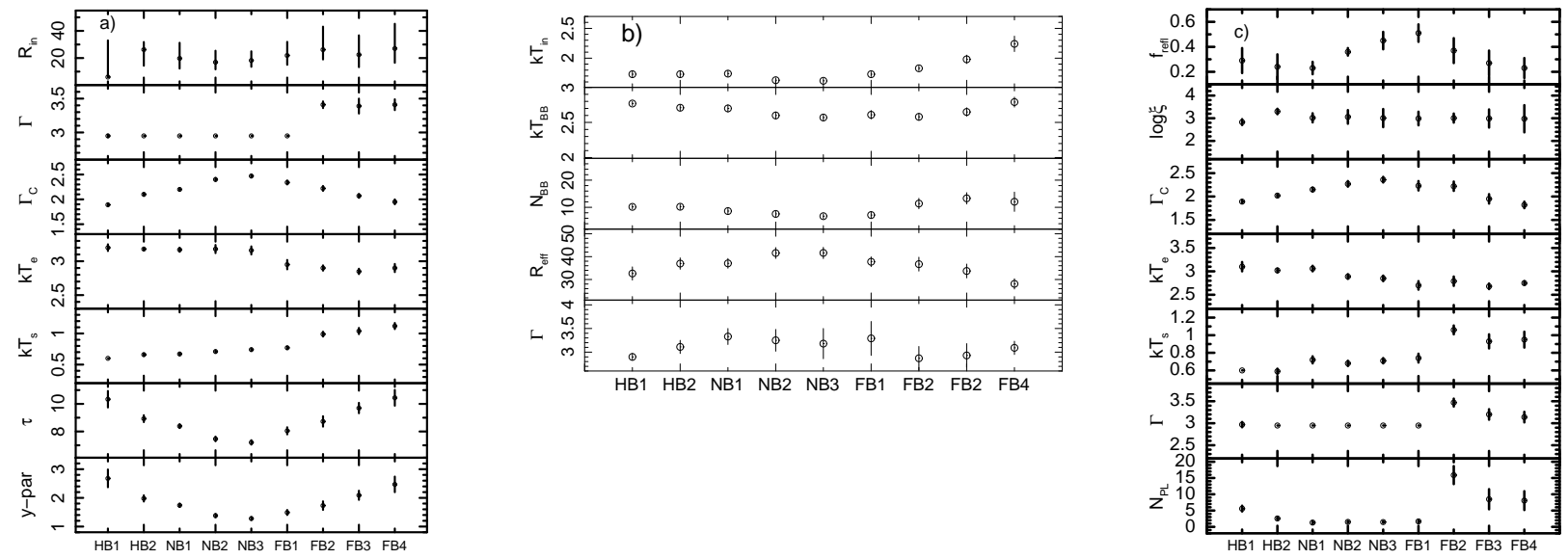

Fig. 5.- Evolution of the best fit spectral parameters along the Z-track of GX 17+2 a) for Model 1, b) for Model 2, and c) for Model 3. See text, Table 1, Table 3 and Table 4 for details. 
model. We find that Model 3 provides comparable and even better fit (for some section of the Z-track) compared to Model 1 and Model 2 (see Table 4). In order to compare our results with previous work (Ludham et al. 2017), we also aply BBREFL (Ballantyne 2004) convolved with RELCONV (Dauser et al. 2010) which describe the reflection of blackbody component from the accretion disc. We find that diskbb+relconv*bbrefl+power-law model successfully describes the spectra at different parts of Z-track (see Table 5). We refer to this model as Model 4. While fitting the spectra with this model we allow the inclination to vary between $25^{\circ}-40^{\circ}$. The inclination peges to the lower limit. We also note that if we keep the inclination angle and the inner disk radius as free parameters, the inner disk radius is not constrained. Hence, we fix the inclination at the lower limit $25^{\circ}$. The neutral hydrogen column density is fixed to $2 \times 10^{22} \mathrm{~cm}^{-2}$ (Di Salvo et al. 2000) in all the four models described above.

\section{3. $\quad$ Timing Analysis}

First, we generate the lightcurves with a binsize of 0.4 milli seconds in the $3.0-60.0 \mathrm{keV}$ band. Lightcurves are divided into intervals of 32768 bins. For each interval, we create the PDS. The PDS corresponding to the same section of the HID are averaged and then rebinned in the frequency space. The binned PDS are normalized to the fractional rms spectra (in units of $(\mathrm{rms} / \text { mean })^{2} \mathrm{~Hz}^{-1}$ ). Dead time corrected Poisson noise level (see Zhang et al. 1995; Agrawal et al. 2018) is subtracted from all the PDS. Since power is not significant above 100 $\mathrm{Hz}$, we use only $0.07-100 \mathrm{~Hz}$ frequency range for the fitting. The PDS is fitted with the combinations of a power-law and/or Lorentzian functions. We find two types of noise features in the PDS: a very low frequency noise (VLFN) modelled using power-law function $\left(A \nu^{-\alpha}\right)$ with index $\alpha$ and normalization $A$ and band limited noise (BLN) component modelled using Lorentzian function defined as,

$$
F_{\nu}=L N /\left(1+\left(2\left(\nu-\nu_{L}\right) / F W H M\right)^{2}\right),
$$

where $L N$ is the normalization, $\nu_{L}$ is the centroid frequency and $F W H M$ is full-widthhalf-maximum of the Lorentzian. In this representation, $\pi * L N * F W H M / 2$ gives the integral of Lorentzian from 0 to $\infty$. The quality factor (Q) of the Lorentzian feature is defined as $\nu_{L} / F W H M$. If BLN centroid frequency is $>10 \mathrm{~Hz}$, then we call it high-frequency noise (HFN) otherwise we call it low frequency noise (LFN). The narrow feature with $Q>3$ is called QPO. Details of the power spectral components observed in the different branches of the Z-track are summarized in Table 6. 
Table 1: The best fit model parameters of Model 1. $k T_{e}$ is the electron temperature, $k T_{s}$ is the seed photon temperature in $\mathrm{keV}, \Gamma_{C}$ is the photon index and $N_{\text {Comp }}$ is the normalization of the $n$ thComp component. $\tau$ is the optical depth and $y$-par is the Comptonization parameter. $E_{L}$ is the line energy, $R_{i n}$ is the inner disc radius and $N_{L}$ is the normalization of the diskline component. $\Gamma$ is the photon index and $N_{P L}$ is the normalization of power-law. $N_{P L}$ is in units of photons $/ \mathrm{s} / \mathrm{cm}^{2} / \mathrm{keV}$ at $1 \mathrm{keV} . N_{L}$ is the line flux in units of photons $/ \mathrm{s} / \mathrm{cm}^{2}$. F-test Prob is the chance improvement probability for an inclusion of a power-law. $F_{\text {Comp }}$ is the $3-80 \mathrm{keV}$ th Comp unabsorbed flux in units of ergs $/ \mathrm{s} / \mathrm{cm}^{2}$ and $F_{P L}$ is the unabsorbed power-law flux in $3-80 \mathrm{keV}$ band in units of $\mathrm{ergs} / \mathrm{s} / \mathrm{cm}^{2}$.

\begin{tabular}{|c|c|c|c|c|c|c|c|c|c|}
\hline 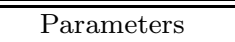 & $\overline{\mathrm{HB} 1}$ & $\overline{\mathrm{HB} 2}$ & $\overline{\mathrm{NB} 1}$ & $\overline{\mathrm{NB} 2}$ & NB3 & FB1 & FB2 & FB3 & FB4 \\
\hline$k T_{e}(\mathrm{keV})$ & $3.20 \pm 0.05$ & $3.18 \pm 0.02$ & $3.17 \pm 0.03$ & $3.18 \pm 0.06$ & $3.16 \pm 0.06$ & $2.95 \pm 0.07$ & $2.90 \pm 0.04$ & $2.85 \pm 0.04$ & $2.90 \pm 0.06$ \\
\hline$\Gamma_{C}$ & $1.89 \pm 0.03$ & $2.10 \pm 0.03$ & $2.20 \pm 0.02$ & $2.40 \pm 0.03$ & $2.47 \pm 0.03$ & $2.34 \pm 0.04$ & $2.22 \pm 0.05$ & $2.07 \pm 0.04$ & $1.95 \pm 0.05$ \\
\hline$k T_{s}(\mathrm{keV})$ & 0.6 (fixed) & $0.66 \pm 0.02$ & $0.67 \pm 0.02$ & $0.71 \pm 0.02$ & $0.74 \pm 0.02$ & $0.77 \pm 0.02$ & $0.99 \pm 0.04$ & $1.04 \pm 0.05$ & $1.12 \pm 0.05$ \\
\hline$N_{\text {Comp }}$ & $0.90 \pm 0.08$ & $1.09 \pm 0.03$ & $1.11 \pm 0.05$ & $1.01 \pm 0.04$ & $0.88 \pm 0.05$ & $0.80 \pm 0.04$ & $0.49 \pm 0.05$ & $0.50 \pm 0.04$ & $0.45 \pm 0.03$ \\
\hline$\tau$ & $10.35 \pm 0.61$ & $8.93 \pm 0.25$ & $8.38 \pm 0.14$ & $7.46 \pm 0.17$ & $7.21 \pm 0.16$ & $8.05 \pm 0.26$ & $8.73 \pm 0.38$ & $9.71 \pm 0.38$ & $10.44 \pm 0.57$ \\
\hline$y-p a r$ & $2.68 \pm 0.31$ & $1.98 \pm 0.11$ & $1.75 \pm 0.06$ & $1.38 \pm 0.06$ & $1.28 \pm 0.05$ & $1.49 \pm 0.09$ & $1.73 \pm 0.15$ & $2.09 \pm 0.16$ & $2.48 \pm 0.27$ \\
\hline$R_{W}(\mathrm{~km})$ & $70.3 \pm 2.4$ & $71.7 \pm 4.3$ & $71.4 \pm 6.3$ & $63.8 \pm 5.4$ & $57.2 \pm 4.6$ & $51.6 \pm 5.3$ & $30.8 \pm 2.4$ & $28.9 \pm 2.7$ & $24.8 \pm 2.2$ \\
\hline$E_{L}(\mathrm{keV})$ & $6.79 \pm 0.08$ & $6.91 \pm 0.05$ & $6.95 \pm 0.07$ & $6.98 \pm 0.06$ & $6.92 \pm 0.04$ & $6.94 \pm 0.06$ & $6.89 \pm 0.07$ & $6.92 \pm 0.08$ & $6.89 \pm 0.09$ \\
\hline$R_{i n}\left(G M / c^{2}\right)$ & $<33$ & $26.25_{-11.92}^{+5.56}$ & $19.76_{-7.51}^{+11.51}$ & $16.90_{-5.21}^{+8.51}$ & $18.22_{-4.67}^{+6.78}$ & $21.91_{-6.88}^{+10.05}$ & $26.18_{-7.22}^{+15.97}$ & $22.51_{-9.21}^{+14.25}$ & $27.01_{-10.51}^{18.27}$ \\
\hline$N_{L}\left(\times 10^{-2}\right)$ & $1.89_{-0.6}^{+0.12}$ & $1.37_{-0.13}^{+0.23}$ & $1.43_{-0.17}^{+0.26}$ & $1.76_{-0.17}^{+0.15}$ & $1.95_{-0.16}^{+0.23}$ & $2.16_{-0.22}^{+0.31}$ & $2.36_{-0.35}^{+0.23}$ & $\begin{array}{r}2.54 \pm 0.31 \\
2.31\end{array}$ & $2.29 \pm 0.20$ \\
\hline$E W(\mathrm{eV})$ & $171.91_{-54.25}^{+10.92}$ & $117.10_{-11.13}^{+19.66}$ & $128.62_{-15.29}^{+23.38}$ & $182.32_{-17.61}^{+15.54}$ & $222.34_{-18.25}^{+26.23}$ & $211.55_{-15.68}^{+30.37}$ & $166.54_{-24.70}^{+16.23}$ & $143.50 \pm 17.52$ & $116.41 \pm 20.33$ \\
\hline$\Gamma$ & $2.95 \pm 0.02$ & 2.95 (fixed) & 2.95 (fixed) & 2.95 (fixed) & 2.95 (fixed) & 2.95 (fixed) & $3.41 \pm 0.05$ & $3.39 \pm 0.11$ & $3.41 \pm 0.08$ \\
\hline$N_{P L}$ & $4.65_{-1.55}^{+0.75}$ & $1.47 \pm 0.16$ & $0.67 \pm 0.51$ & $0.67 \pm 0.49$ & $0.79 \pm 0.44$ & $1.08 \pm 0.41$ & $10.81_{-2.12}^{+3.15}$ & $12.35_{-1.39}^{+3.04}$ & $15.50_{-2.53}^{1.94}$ \\
\hline$F_{C o m p}\left(\times 10^{-9}\right)$ & $13.18 \pm 0.31$ & $14.46 \pm 0.33$ & $14.12 \pm 0.31$ & $12.35 \pm 0.28$ & $10.97 \pm 0.25$ & $11.76 \pm 0.27$ & $\begin{array}{l}14.14 \pm 0.33 \\
-2.12\end{array}$ & $\begin{array}{l}17.79 \pm 0.41\end{array}$ & $\begin{array}{l}20.42 \pm 0.47 \\
20.43\end{array}$ \\
\hline$F_{P L}\left(\times 10^{-9}\right)$ & $2.63_{-0.5}^{+0.6}$ & $0.93_{-0.24}^{+0.34}$ & $0.49_{-0.23}^{+0.31}$ & $\begin{array}{l}0.41_{-0.21}^{+0.30} \\
0.00\end{array}$ & $0.44_{-0.24}^{+0.21}$ & $0.59_{-0.27}^{+0.36}$ & $\begin{array}{l}14.14 \pm 0.00 \\
2.58_{-0.26}^{+0.31}\end{array}$ & $\begin{array}{l}1.1 .0 .41 \\
3.09_{-0.33}^{+0.37}\end{array}$ & $\begin{array}{l}20.42 \pm 0.41 \\
3.70_{-0.32}^{+0.35}\end{array}$ \\
\hline$F_{t o t}\left(\times 10^{-9}\right)$ & $15.82 \pm 0.8$ & $15.38 \pm 0.51$ & $14.59 \pm 0.42$ & $12.71 \pm 0.48$ & $11.41 \pm 0.34$ & $12.33 \pm 0.44$ & $16.66 \pm 0.45$ & $20.8 \pm 0.55$ & $24.15 \pm 0.58$ \\
\hline$F_{P L}(\%)$ & 16.6 & 6.08 & 3.36 & 3.20 & 3.82 & 4.77 & 15.48 & 14.80 & 15.32 \\
\hline$\chi^{2} /$ dof & $67 / 79$ & $65 / 79$ & $78 / 79$ & $90 / 79$ & $88 / 79$ & $101 / 79$ & $85 / 78$ & $78 / 78$ & $67 / 78$ \\
\hline$F-$ testProb. & $4.4 \mathrm{e}-9$ & $1.3 e-5$ & $2.7 \mathrm{e}-02$ & $6.2 \mathrm{e}-02$ & $6.6 \mathrm{e}-02$ & $3.4 \mathrm{e}-02$ & $3.5 \mathrm{e}-09$ & $6.8 \mathrm{e}-10$ & $2.2 \mathrm{e}-14$ \\
\hline
\end{tabular}


Table 2: The best fit spectral parameters of Model 1 with a Gaussian instead of a diskline for the iron line. $E_{F e}$ is line energy for Gaussian line in units of $\mathrm{keV}, \sigma_{F e}$ is the width of line in units of $\mathrm{keV}$ and $N_{F e}$ is the normalization of the line in units of photons $/ \mathrm{s} / \mathrm{cm}^{2}$. Other paramters are same as in Table 1.

\begin{tabular}{|c|c|c|c|c|c|c|c|c|c|}
\hline Parameters & HB1 & HB2 & NB1 & NB2 & NB3 & FB1 & FB2 & FB3 & FB4 \\
\hline$\Gamma$ & $1.84 \pm 0.05$ & $2.06 \pm 0.02$ & $2.14 \pm 0.03$ & $2.32 \pm 0.04$ & $2.35 \pm 0.05$ & $2.20 \pm 0.02$ & $2.02 \pm 0.06$ & $1.91 \pm 0.07$ & $1.85 \pm 0.07$ \\
\hline$k T_{e}$ & $3.15 \pm 0.07$ & $3.12 \pm 0.05$ & $3.12 \pm 0.06$ & $3.13 \pm 0.08$ & $3.02 \pm 0.07$ & $2.83 \pm 0.05$ & $2.73 \pm 0.07$ & $2.72 \pm 0.06$ & $2.81 \pm 0.06$ \\
\hline$k T_{s}$ & 0.6(fixed) & $0.59 \pm 0.04$ & $0.61 \pm 0.04$ & $0.64 \pm 0.04$ & $0.64 \pm 0.05$ & $0.63 \pm 0.06$ & $0.83 \pm 0.12$ & $0.87 \pm 0.12$ & $1.02 \pm .09$ \\
\hline$\Gamma_{P L}$ & $3.06 \pm 0.06$ & 2.95(fixed) & 2.95(fixed) & 2.95(fixed) & 2.95(fixed) & 2.95(fixed) & $3.25 \pm 0.19$ & $3.22 \pm 0.18$ & $3.35 \pm 0.10$ \\
\hline$N_{P L}$ & $6.95 \pm 1.2$ & $1.81 \pm 0.51$ & $0.80 \pm 0.52$ & $0.63 \pm 0.48$ & $0.95 \pm 0.45$ & $1.19 \pm 0.42$ & $8.15 \pm 3.2$ & $8.67 \pm 3.3$ & $14.75 \pm 3.2$ \\
\hline$E_{F e}(\mathrm{keV})$ & $6.4 \pm 0.49$ & $6.57 \pm 0.21$ & $6.52 \pm 0.18$ & $6.45 \pm 0.21$ & $6.43 \pm 0.35$ & $6.48 \pm 0.17$ & $6.61 \pm 0.18$ & $6.69 \pm 0.28$ & $6.54 \pm 0.29$ \\
\hline$\sigma_{F e}(\mathrm{keV})$ & $0.99 \pm 0.24$ & $0.92 \pm 0.20$ & $1.05 \pm 0.22$ & $1.09 \pm 0.16$ & $1.04 \pm 0.27$ & $1.06 \pm 0.2$ & $0.95 \pm 0.18$ & $0.95 \pm 0.29$ & $1.20 \pm 0.22$ \\
\hline$N_{F e}\left(\times 10^{-2}\right)$ & $2.82 \pm 0.81$ & $2.59 \pm 0.72$ & $3.01 \pm 1.1$ & $3.98 \pm 1.1$ & $4.1 \pm 1.2$ & $4.86 \pm 0.7$ & $4.68 \pm 1.4$ & $4.85 \pm 0.21$ & $6.95 \pm 2.71$ \\
\hline$\chi^{2} / d o f$ & $66 / 79$ & $61 / 79$ & $75 / 79$ & $85 / 79$ & $73 / 79$ & $103 / 79$ & $79 / 78$ & $76 / 78$ & $69 / 78$ \\
\hline
\end{tabular}


Table 3: Best fit parameters for Model 2. $k T_{i n}$ is the inner disk temperature, $N_{d b b}$ is the normalization of the diskbb, $R_{e f f}$ is the effective radius of inner disk, $k T_{B B}$ is the blackbody temperature and $N_{B B}$ is the normalization of the blackbody component. F-test Prob. is the chance improvement probability for an inclusion of the power-law

\begin{tabular}{|c|c|c|c|c|c|c|c|c|c|}
\hline Parameters & HB1 & HB2 & NB1 & NB2 & NB3 & FB1 & FB2 & FB3 & FB4 \\
\hline$k T_{i n}(\mathrm{keV})$ & $1.73 \pm 0.05$ & $1.73 \pm 0.05$ & $1.74 \pm 0.05$ & $1.63 \pm 0.04$ & $1.62 \pm 0.04$ & $1.73 \pm 0.04$ & $1.83 \pm 0.04$ & $1.98 \pm 0.06$ & $2.24 \pm 0.12$ \\
\hline$N_{d b b}$ & $59.71 \pm 10.55$ & $76.51 \pm 10.35$ & $76.96 \pm 8.95$ & $96.85 \pm 11.15$ & $97.02 \pm 11.45$ & $79.85 \pm 9.05$ & $75.55 \pm 12.5$ & $63.82 \pm 11.45$ & $44.25 \pm 7.15$ \\
\hline$R_{e f f}($ in $\mathrm{km})$ & $32.65 \pm 2.83$ & $36.98 \pm 2.48$ & $37.05 \pm 2.15$ & $41.58 \pm 2.39$ & $41.64 \pm 2.45$ & $37.75 \pm 2.13$ & $36.68 \pm 2.03$ & $33.75 \pm 3.02$ & $28.10 \pm 2.26$ \\
\hline$k T_{B B}(\mathrm{keV})$ & $2.77 \pm 0.03$ & $2.71 \pm 0.04$ & $2.70 \pm 0.05$ & $2.60 \pm 0.05$ & $2.57 \pm 0.04$ & $2.61 \pm 0.06$ & $2.58 \pm 0.05$ & $2.65 \pm 0.05$ & $2.79 \pm 0.07$ \\
\hline$N_{B B}$ & $10.19 \pm 0.85$ & $10.21 \pm 0.98$ & $8.65 \pm 0.95$ & $7.61 \pm 0.95$ & $6.75 \pm 0.93$ & $7.19 \pm 1.23$ & $11.38 \pm 1.92$ & $13.32 \pm 2.05$ & $12.06 \pm 3.55$ \\
\hline$R_{B B}(\mathrm{~km})$ & $4.2 \pm 0.1$ & $4.2 \pm 0.1$ & $3.8 \pm 0.2$ & $3.6 \pm 0.2$ & $3.4 \pm 0.2$ & $3.5 \pm 0.3$ & $4.4 \pm 0.3$ & $4.7 \pm 0.3$ & $4.5 \pm 0.6$ \\
\hline$R_{\text {Beff }}(\mathrm{km})$ & $9.3 \pm 0.3$ & $9.3 \pm 0.2$ & $8.55 \pm 0.5$ & $8.05 \pm 0.5$ & $7.5 \pm 0.5$ & $7.8 \pm 0.7$ & $9.8 \pm 0.8$ & $10.6 \pm 0.7$ & $10.2 \pm 1.1$ \\
\hline$\Gamma_{P L}$ & $2.90 \pm 0.07$ & $3.11 \pm 0.15$ & $3.33 \pm 0.17$ & $3.25 \pm 0.23$ & $3.18 \pm 0.32$ & $3.29 \pm 0.36$ & $2.87 \pm 0.25$ & $2.93 \pm 0.25$ & $3.09 \pm 0.14$ \\
\hline$N_{P L}$ & $5.22 \pm 1.17$ & $4.86 \pm 1.44$ & $5.33 \pm 1.68$ & $3.52 \pm 1.95$ & $2.49 \pm 1.85$ & $2.56 \pm 2.49$ & $1.96 \pm 1.08$ & $2.64 \pm 1.48$ & $3.09 \pm 4.78$ \\
\hline$E_{L}$ & $6.87 \pm 0.87$ & $6.89 \pm 0.11$ & $6.86 \pm 0.12$ & $6.93 \pm 0.08$ & $6.87 \pm 0.06$ & $6.86 \pm 0.06$ & $6.89 \pm 0.04$ & $6.91 \pm 0.07$ & $6.89 \pm 0.07$ \\
\hline$R_{i n}\left(G M / c^{2}\right)$ & $39.85_{-20}^{+60}$ & $40.95_{-22}^{+75}$ & $39.5_{-21}^{+60}$ & $21.6_{-12}^{+17}$ & $20.78_{-9.8}^{+15}$ & $24.56_{-9.8}^{+24}$ & $24.75_{-9.8}^{+22}$ & $20.81_{-9.4}^{+16}$ & $21.37_{-9.4}^{+20}$ \\
\hline$N_{L}\left(\times 10^{-2}\right)$ & $1.17 \pm 0.14$ & $1.09 \pm 0.15$ & $1.04 \pm 0.10$ & $1.44 \pm 0.20$ & $1.69 \pm 0.21$ & $1.99 \pm 0.27$ & $2.41 \pm 0.27$ & $2.69 \pm 0.37$ & $2.61 \pm 0.28$ \\
\hline$F_{\text {total }}\left(\times 10^{-9}\right)$ & $15.71 \pm 0.39$ & $15.40 \pm 0.43$ & $14.29 \pm 0.43$ & $12.25 \pm 0.38$ & $11.17 \pm 0.41$ & $12.32 \pm 0.41$ & $16.82 \pm 0.72$ & $20.8 \pm 0.95$ & $24.31 \pm 0.93$ \\
\hline$F_{P L}\left(\times 10-{ }^{9}\right)$ & $3.23 \pm 0.23$ & $2.02 \pm 0.29$ & $1.36 \pm 0.32$ & $1.12 \pm 0.29$ & $0.90 \pm 0.34$ & $0.75 \pm 0.33$ & $1.35 \pm 0.38$ & $1.51 \pm 0.39$ & $2.09 \pm 0.30$ \\
\hline$F_{P L}($ in $\%)$ & 20.59 & 13.10 & 9.51 & 9.12 & 8.05 & 6.08 & 8.02 & 7.25 & 8.60 \\
\hline$\chi^{2} / d o f$ & $63 / 78$ & $62 / 78$ & $77 / 78$ & $81 / 78$ & $81 / 78$ & $113 / 78$ & $85 / 78$ & $80 / 78$ & $70 / 78$ \\
\hline$F-$ test Prob. & $4.7 \mathrm{e}-22$ & $1.2 \mathrm{e}-14$ & $9.1 \mathrm{e}-7$ & $1.8 \mathrm{e}-4$ & $3.0 \mathrm{e}-3$ & $6.9 \mathrm{e}-2$ & $7.5 \mathrm{e}-6$ & $1.1 \mathrm{e}-5$ & $2.2 \mathrm{e}-8$ \\
\hline
\end{tabular}


Table 4: Best fit parameters for Model 3. $f_{r e f l}$ is reflection fraction, $A_{F e}$ is iron abundance and $\log \xi$ is the logarithm of ionization parameter.

\begin{tabular}{cccccccccc}
\hline \hline Parameters & HB1 & HB2 & NB1 & NB2 & NB3 & FB1 & FB2 & FB3 & FB4 \\
\hline$f_{r e f l}$ & $0.29 \pm 0.11$ & $0.24 \pm 0.09$ & $0.23 \pm 0.04$ & $0.36 \pm 0.04$ & $0.45 \pm 0.07$ & $0.51 \pm 0.07$ & $0.37 \pm 0.09$ & $0.27 \pm 0.09$ & $0.23 \pm 0.08$ \\
$A_{F e}$ & $0.49 \pm 0.23$ & 0.5 (fixed) & 0.5 (fixed) & 0.5 (fixed) & 0.5 (fixed) & $0.49 \pm 0.31$ & 0.5 (fixed) & 0.5 (fixed) & 0.5 (fixed) \\
$\log \xi$ & $2.83 \pm 0.13$ & $3.30 \pm 0.12$ & $3.02 \pm 0.21$ & $3.06 \pm 0.31$ & $3.01 \pm 0.4$ & $2.99 \pm 0.31$ & $3.01 \pm 0.22$ & $2.99 \pm 0.38$ & $2.98 \pm 0.55$ \\
$\Gamma_{C}$ & $1.89 \pm 0.03$ & $2.02 \pm 0.03$ & $2.15 \pm 0.04$ & $2.27 \pm 0.05$ & $2.36 \pm 0.04$ & $2.23 \pm 0.03$ & $2.22 \pm 0.09$ & $1.95 \pm 0.11$ & $1.82 \pm 0.07$ \\
$k T_{e}(\mathrm{keV})$ & $3.10 \pm 0.08$ & $3.02 \pm 0.04$ & $3.06 \pm 0.06$ & $2.89 \pm 0.06$ & $2.85 \pm 0.05$ & $2.70 \pm 0.09$ & $2.79 \pm 0.08$ & $2.68 \pm 0.06$ & $2.75 \pm 0.04$ \\
$k T_{s}(\mathrm{keV})$ & 0.6 (fixed) & $0.59 \pm 0.03$ & $0.72 \pm 0.05$ & $0.68 \pm 0.03$ & $0.71 \pm 0.03$ & $0.74 \pm 0.05$ & $1.06 \pm 0.05$ & $0.93 \pm 0.08$ & $0.95 \pm 0.09$ \\
$\Gamma_{P L}$ & $2.96 \pm 0.06$ & 2.95 (fixed) & $2.95($ fixed) & $2.95($ fixed) & $2.95($ fixed) & $2.95($ fixed) & $3.47 \pm 0.09$ & $3.20 \pm 0.12$ & $3.14 \pm 0.12$ \\
$N_{P L}$ & $5.60 \pm 0.86$ & $2.56 \pm 0.4$ & $1.30 \pm 0.41$ & $1.49 \pm 0.1$ & $1.46 \pm 0.25$ & $1.65 \pm 0.36$ & $15.88 \pm 2.77$ & $8.48 \pm 3.1$ & $8.07 \pm 2.9$ \\
$\chi^{2} /$ dof & $56 / 79$ & $61 / 80$ & $72 / 80$ & $78 / 80$ & $68 / 80$ & $102 / 79$ & $72 / 79$ & $81 / 79$ & $74 / 79$ \\
\hline \hline
\end{tabular}


Table 5: Best fit parameters for Model 4. $f_{\text {refl }}$ is reflection fraction, iron abundance is fixed 1.0. $\log \xi$ is the logarithm of ionization parameter. $k T_{B B}$ and $k T_{i n}$ is the blackbody and inner disk temperature (in keV) respectively. The parameter norm is BBREFL normalization. $\Gamma$ and $N_{P L}$ are index and normalization of the power-law component.

\begin{tabular}{cccccccccc}
\hline \hline Parameters & HB1 & HB2 & NB1 & NB2 & NB3 & FB1 & FB2 & FB3 & FB4 \\
\hline$R_{\text {in }}$ (ISCO) & $14.02 \pm 5.4$ & $18.5 \pm 4.2$ & $18.6 \pm 3.9$ & $15.4 \pm 4.3$ & $13.3 \pm 4.1$ & $14.4 \pm 3.9$ & $13.2 \pm 3.7$ & $12.35 \pm 4.8$ & $12.45 \pm 4.4$ \\
$\log \xi$ & $2.64 \pm 0.5$ & $2.66 \pm 0.4$ & $2.68 \pm 0.45$ & $2.81 \pm 0.4$ & $\mathbf{2 . 7 2} \pm \mathbf{0 . 2}$ & $2.71 \pm 0.3$ & $2.73 \pm 0.4$ & $2.64 \pm 0.3$ & $2.73 \pm 0.3$ \\
$f_{\text {refl }}$ & $0.36 \pm 0.14$ & $0.34 \pm 0.12$ & $0.35 \pm 0.12$ & $0.61 \pm 0.06$ & $0.88 \pm 0.11$ & $0.86 \pm 0.10$ & $0.76 \pm 0.22$ & $0.72 \pm 0.32$ & $0.67 \pm 0.45$ \\
$n$ orm $\left(1 \times 10^{-26}\right)$ & $17.5 \pm 7.6$ & $15.9 \pm 4.4$ & $10.6 \pm 5.2$ & $6.8 \pm 1.9$ & $6.4 \pm 2.5$ & $7.23 \pm 3.5$ & $10.8 \pm 4.5$ & $17.4 \pm 5.5$ & $14.2 \pm 4.9$ \\
$k T_{B B}(\mathrm{keV})$ & $2.65 \pm 0.03$ & $2.54 \pm 0.03$ & $2.50 \pm 0.04$ & $2.39 \pm 0.02$ & $2.35 \pm 0.04$ & $2.35 \pm 0.05$ & $2.42 \pm 0.06$ & $2.52 \pm 0.15$ & $2.69 \pm 0.22$ \\
$k T_{\text {in }}(\mathrm{keV})$ & $1.64 \pm 0.10$ & $1.60 \pm 0.05$ & $1.59 \pm 0.05$ & $\mathbf{1 . 5 0} \pm \mathbf{0 . 0 4}$ & $1.51 \pm 0.06$ & $1.58 \pm 0.06$ & $1.77 \pm 0.07$ & $1.99 \pm 0.25$ & $2.29 \pm 0.5$ \\
$N_{\text {dbb }}$ & $70.5 \pm 20.5$ & $104.4 \pm 13.6$ & $111.6 \pm 12.2$ & $127.8 \pm 14.3$ & $122.7 \pm 12.4$ & $104.3 \pm 15.5$ & $76.5 \pm 19.4$ & $56.8 \pm 25.3$ & $36.7 \pm 17.9$ \\
$\Gamma$ & $2.85 \pm 0.06$ & $2.90(\mathrm{fix})$ & $2.90(\mathrm{fix})$ & $2.90(\mathrm{fix})$ & $2.90(\mathrm{fix})$ & $2.90(\mathrm{fix})$ & $3.01 \pm 0.15$ & $3.04 \pm 0.18$ & $3.15 \pm 0.13$ \\
$N_{P L}$ & $5.41 \pm 0.91$ & $2.93 \pm 0.45$ & $2.03 \pm 0.35$ & $2.05 \pm 0.36$ & $1.90 \pm 0.34$ & $1.91 \pm 0.38$ & $3.99 \pm 0.85$ & $4.76 \pm 1.25$ & $7.55 \pm 1.32$ \\
$\chi^{2} /$ dof & $57 / 78$ & $60 / 79$ & $73 / 79$ & $67 / 79$ & $59 / 79$ & $95 / 79$ & $65 / 78$ & $68 / 78$ & $56 / 78$ \\
\hline
\end{tabular}



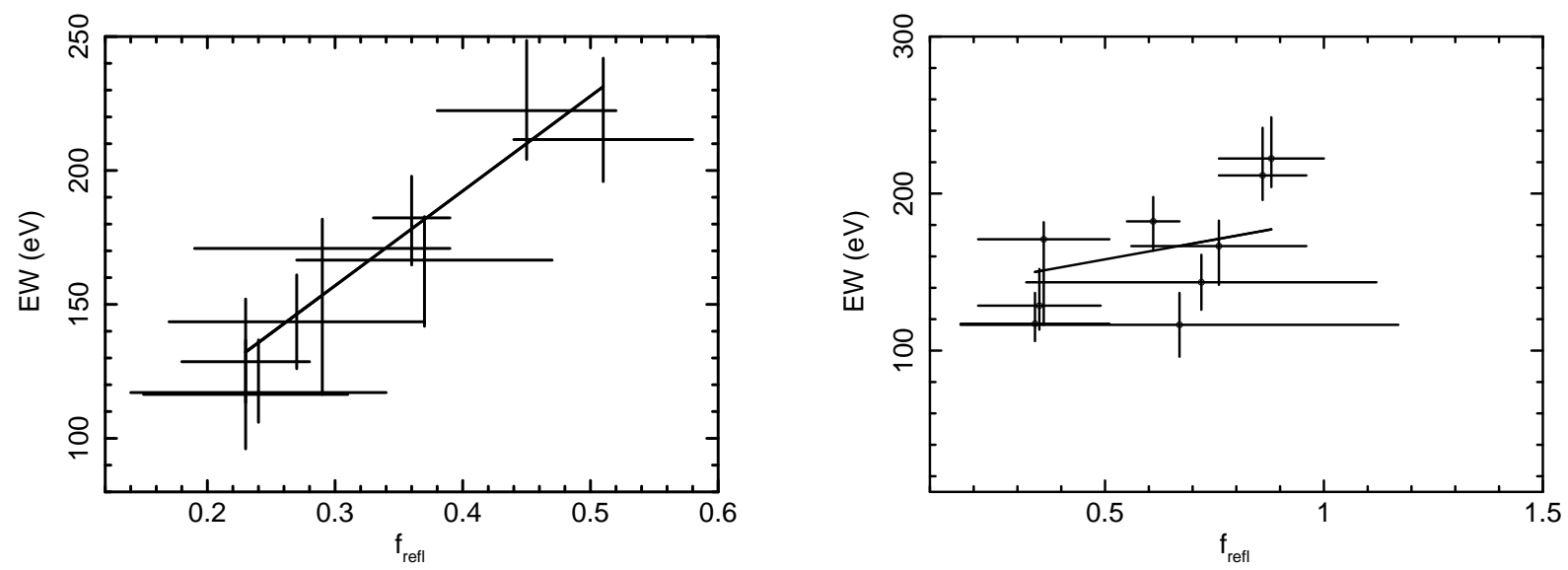

Fig. 6. - Left: Correlation between the equivalent width of the iron line ( Model 1) and the reflection fraction (from Model 3). Right: Correlation between the equivalent width of the iron line (Model 1) and the reflection fraction (from Model 4)

Table 6: Model components of PDS in the frequency range $0.07-100 \mathrm{~Hz}$. Three noise features LFN (Low-frequency noise), HFN (High-frequency noise) and VLFN (Very lowfrequency noise) are observed (in the bracket). The QPO is observed in the NB2 (in the bracket).

\begin{tabular}{cc}
\hline Sections & PDS Model Components \\
\hline HB1 & Lorentzian (LFN) + Lorentzian (HFN) \\
HB2 & Lorentzian (LFN) \\
NB1 & Lorentzian (LFN) \\
NB2 & Power-law (VLFN) + Lorentzian (QPO) \\
NB3 & Power-law (VLFN) + Lorentzian (LFN) \\
FB1 & Lorentzian (LFN) \\
FB2 & Power-law (VLFN) + Lorentzian (LFN) \\
FB3 & Power-law (VLFN) \\
FB4 & Power-law (VLFN) \\
\hline \hline
\end{tabular}


Table 7: The best fit results obtained by fitting the PDS at different parts of the Z-track. $\nu_{L}$ is the centroid frequency, $F W H M$ is the full-width-half-maximum, $L N$ is the normalization of Lorentzian feature. rms is the integrated rms in the $0.07-100 \mathrm{~Hz} . \alpha$ is the index and $A$ is the normalization of the power-law component.

\begin{tabular}{|c|c|c|c|c|c|c|c|c|c|}
\hline Parameters & HB1 & HB2 & NB1 & NB2 & NB3 & FB1 & FB2 & FB3 & FB4 \\
\hline \multicolumn{10}{|c|}{$\overline{\text { LFN (Lorentzian) }}$} \\
\hline$\nu_{L}(H z)$ & $2.53 \pm 0.26$ & 0.0 (fixed) & $3.32 \pm 1.07$ & $7.42 \pm 0.23$ & $8.09 \pm 0.38$ & $6.25 \pm 4.16$ & $7.88 \pm 1.58$ & & \\
\hline$F W H M(H z)$ & $7.20 \pm 1.05$ & $9.16 \pm 0.95$ & $8.96 \pm 4.59$ & $1.52 \pm 0.59$ & $7.13 \pm 1.12$ & $31.02 \pm 9.72$ & $12.29 \pm 3.47$ & & \\
\hline$L N\left(\times 10^{5}\right)$ & $20.42 \pm 1.32$ & $13.93 \pm 1.25$ & $2.56 \pm 0.68$ & $8.47 \pm 1.89$ & $13.12 \pm 1.24$ & $3.75 \pm 0.78$ & $3.22 \pm 0.56$ & & \\
\hline$r m s(\%)$ & $3.96 \pm 0.46$ & $3.11 \pm 0.44$ & $1.58 \pm 0.65$ & $1.41 \pm 0.29$ & $3.54 \pm 0.37$ & $3.22 \pm 1.06$ & $2.18 \pm 0.47$ & & \\
\hline \multicolumn{10}{|c|}{ HFN (Lorentzian) } \\
\hline$\nu_{L}(H z)$ & $25.68 \pm 3.52$ & & & & & & & & \\
\hline$F W H M(H z)$ & $22.44 \pm 7.33$ & & & & & & & & \\
\hline$L N\left(\times 10^{5}\right)$ & $2.34 \pm 0.61$ & & & & & & & & \\
\hline$r m s(\%)$ & $2.59 \pm 0.65$ & & & & & & & & \\
\hline \multicolumn{10}{|c|}{ VLFN (Power-law) } \\
\hline$\alpha$ & & & & $-0.48 \pm 0.21$ & $-1.01 \pm 0.22$ & & $-1.81 \pm 0.26$ & $-1.55 \pm 0.25$ & $-1.45 \pm 0.29$ \\
\hline$A\left(\times 10^{5}\right)$ & & & & $1.20 \pm 0.69$ & $2.55 \pm 1.33$ & & $0.63 \pm 0.24$ & $1.77 \pm 1.12$ & $3.55 \pm 0.95$ \\
\hline$r m s(\%)$ & & & & $1.59 \pm 1.21$ & $1.39 \pm 0.24$ & & $0.94 \pm 0.01$ & $1.29 \pm 0.018$ & $1.72 \pm 0.05$ \\
\hline Total-rms(\%) & $4.73 \pm 0.70$ & $3.11 \pm 0.44$ & $1.58 \pm 0.65$ & $2.12 \pm 1.25$ & $3.80 \pm 0.35$ & $3.22 \pm 1.06$ & $2.38 \pm 0.47$ & $1.32 \pm 0.025$ & $1.72 \pm 0.05$ \\
\hline$\chi^{2} /$ dof & $26 / 54$ & $32 / 57$ & $56 / 57$ & $26 / 55$ & $42 / 55$ & $60 / 57$ & $40 / 55$ & $33 / 58$ & $44 / 58$ \\
\hline
\end{tabular}





Fig. 7.- PDS and the best fit model at three different parts of the Z-track. HB1: top-left panel, NB2: top-right panel and FB2: bottom panel. The used model is described in Table 6 and best fit parameters are given in Table 7. A significant QPO is clearly seen in the PDS of NB2.

\section{Results}

\subsection{Spectral Properties}

The $3.0-80.0 \mathrm{keV}$ X-ray spectra extracted from the different sections of the Z-track can be fitted by two different phenomenological models. Model 1 consists of a Comptonized emission, a relativistic iron line feature and a high energy tail. Table 1 shows the best fit parameters. The F-test chance improvement probability for an inclusion of a power-law tail is also listed in the Table 1. Model 2, which consists of disc emission, a blackbody emission component, a relativistic iron line and a non-thermal tail, is also statistically good description of the data. In Figure 3, we show the unfolded spectra for HB1, NB2 and FB2 fitted with these two models. The best fit parameters of Model $\mathbf{2}$ are shown in the Table 3. We also note that Model $\mathbf{3}$ and Model $\mathbf{4}$ which includes reflection component to explain the origin of iron line is also a good description of the spectra for all the sections of the 
Z-track. In Figure 4a we show the unfolded spectrum of the HB1 section fitted with Model 3 and in Figure 4b we show that fitted with Model 4. In Figure 5a, 5b and 5c, we show the variation of the best fit spectral parameters for Model 1 (left panel), Model 2 (middle panel) and Model 3 (right panel) respectively. The best fit parameters of Model 3 and Model 4 are shown in Table 4 and 5 respectively.

The contribution of the high energy tail to the total $3.0-80.0 \mathrm{keV}$ flux is the highest in the HB1 (see Table 1 and Table 3). It gradually fades as the source moves down the HB and then down the NB. As the source moves up along the FB the power-law flux systematically increases. The two different phenomenological models used here give different values of the power-law flux and the parameters of the power-law. However, trend in the variation of the power-law strength along the Z-track is similar.

Modelling the iron line feature with diskline model allows to constrain the inner radius of the disc. Since error present in the inferred inner disc radius is large, we can not comment much about its evolution along the Z-track. The inner disc radius obtained from Model 1 varies between $\sim 10.0-45.0 R_{g}$ being lowest at the upper HB. However, $R_{i n}$ inferred from the Model 2 is in the range of $\sim 20.0-40.0$. The equivalent width of the iron line is found to be in the range of $\sim 115-220 \mathrm{eV}$. The dominant emission component of Model 1 present in the $3-80 \mathrm{keV}$ spectra is thermal Comptonized emission, modelled with $n$th Comp model of XSPEC. The plasma temperature obtained by fitting with this model is $\sim 3.20$ $\mathrm{keV}$ in the $\mathrm{HB}$ and $\mathrm{NB}$, and $\sim 2.90 \mathrm{keV}$ in the FB. The photon index $\left(\Gamma_{C}\right)$ of Comptonized emission shows systematic evolution along the Z-track (see Figure 5a). The index $\Gamma_{C}$ initially increases as the source moves along the HB and then down the NB. $\Gamma_{C}$ again decreases as the source moves up along the FB. Figure 5a shows the evolution of the best fit spectral parameters as a function of the position on the HID. The optical depth $\tau$ is calculated using formula given in Agrawal et al. (2018) and the Comptonization parameter $y$ is calculated using the formula,

$$
y=\frac{4 k T_{e}}{m_{e} c^{2}} \max \left(\tau, \tau^{2}\right),
$$

where $k T_{e}$ is the electron temperature, $m_{e}$ is the mass of the electron, $\tau$ is the optical depth and $\mathrm{c}$ is the speed of light. The seed photon radius $R_{W}$ of the spherical area emitting the blackbody spectrum is given by (in't Zand et al. 1999),

$$
R_{W}=3 \times 10^{4} D \sqrt{\frac{f_{\text {bol }}}{1+y}} /\left(k T_{s}\right)^{2},
$$

where $k T_{s}$ is the seed photon temperature in $\mathrm{keV}, D$ is the distance to the source in kpc and $f_{\text {bol }}$ is the bolometric $(0.1-100 \mathrm{keV})$ luminosity of the Comptonized component. The 
seed photon radius $R_{W}$ varies from $70 \mathrm{~km}$ to $25 \mathrm{~km}$ as the source moves along the Z-track from the $\mathrm{HB}$ to $\mathrm{FB}$, suggesting that inner disc approaches the central source as the source moves along the Z-track from the HB to FB through NB. This is expected if mass accretion rate is increasing from the $\mathrm{HB}$ to $\mathrm{FB}$.

The optical depth $\tau$ of the corona decreases as the source moves down from the HB to the lowest part of the NB. It again increases as the source moves up along the FB. The Comptonization parameter $(y-p a r)$ decreases as the source moves down the HB and then again towards the lower part of the NB. The increase in $y-p a r$ is observed as the source moves up the FB (see Figure 5a and Table 1).

Fitting the data with Model 2 gives inner-disc temperature in the range of $1.6-2.2$ $\mathrm{keV}$ (see Table 3 ). In the diskbb model, inner disk radius $R_{d}$ is given by $\sqrt{N_{d b b} / \cos \theta} D_{10}$, where $D_{10}$ is distance to the source in $10 \mathrm{kpc}$ and $\theta$ is the inclination angle of the disk. Shimura and Takahara (1995) argue that if the electron scattering dominates the opacity, the local spectrum will significantly deviate from the blackbody and is approximated by a diluted blackbody. The local specific flux for diluted blackbody is given by,

$$
F_{\nu}=\frac{\pi}{f_{c o l}^{4}} B_{\nu}\left(f_{c o l} T_{e f f}\right),
$$

where $f_{c o l}$ is the spectral hardening factor, $T_{\text {eff }}$ is the effective temperature and $B_{\nu}$ is the Planck function. Davis et al. (2005) studied the dependence of $f_{c o l}$ on the luminosities and inclination angle. For an inclination angle of $i=45^{\circ}$ and $L / L_{E d d}=0.3$ they found $f_{c o l}=1.6$. The effective inner disc radius and effective temperature is given by,

$$
R_{e f f}=f_{c o l}^{2} R_{d}, \quad T_{e f f}=k T_{i n} / f_{c o l} .
$$

The estimated effective radius varies between $\sim 28-42 \mathrm{~km}$ for an inclination angle $i=40^{\circ}$ and distance to the source $\mathrm{D}=12.6 \mathrm{kpc}$ (Kuulkers et al. 2003). $R_{\text {eff }}$ increases as the source moves from HB1 to NB3 and then decreases as it moves along the FB. The trend in the variation of $R_{e f f}$ and $R_{W}$ is similar along the FB. However, it is opposite in the $\mathrm{HB}$ and NB. The effective temperature is found to be in the range of $1.0-1.4 \mathrm{keV}$. The blackbody component has temperature in the range of $2.6-2.8 \mathrm{keV}$. The blackbody radius in bbodyrad model is given by,

$$
R_{B B}=\sqrt{N_{B B}} D_{10}
$$

where $R_{B B}$ radius of blackbody emitting surface in units of $\mathrm{km}, N_{B B}$ is normalization of bbodyrad and $D_{10}$ the source distance in the units of $10 \mathrm{kpc}$. The blackbody radius is found to be $\sim 4 \mathrm{~km}$ at different parts of the Z-track.

Foster, Ross and Fabian (1986) fitted a series of numerical models assuming constant density atmosphere to the spectra of type I X-ray bursts observed by EXOSAT. They derived 
relationship between the effective blackbody temperature and the fitted or colour temperature. They found colour correction factor $\left(f_{\text {col }}\right)$ in the range of $\sim 1.3-1.6$. Using the average colour correction factor $f_{\text {col }} \sim 1.5$, we compute the effective blackbody radius $R_{B e f f}$ using equation,

$$
R_{\text {Beff }}=f_{c o l}^{2} R_{B B},
$$

The effective blackbody radius is found to be in the range of $8-10 \mathrm{~km}$ (see Table 3 ).

Model 3 gives iron abundance $A_{F e} \sim 0.5$. The reflection fraction $f_{r e f l}$ varies between $0.2-0.5$ along the Z-track and logarithm of ionization parameter is found to be around 3.0 on the Z-track (see Table 4 and Figure 5c). The X-ray spectrum of the section HB1, fitted with Model 3 is shown in the Figure 4a. We note that the iron line observed in the spectra of the source at different parts of the Z-track is narrow and does not require an additional relativistic smearing. We checked this by introducing additional bluring using the convolution model rdblur and found that the fit is not improved. We also plot the equivalent width of iron line derived using Model 1 against reflection fraction derived using Model 3. A clear correlation between these two parameters can be seen (see Figure 6). Model 4 gives reflection fraction $f_{\text {refl }}$ in the range of $0.3-0.9$. It also suggests that the iron line is narrow and disc is truncated away from the central compact object $\left(10-22 R_{I S C O}\right)$. The logrithm of ionization parameter is found to be around $\sim 2.5-3.0$. In Model 4 , we have fixed the iron abundance at 1.0 .

\subsection{Timing Behaviour}

The timing analysis of the source revealed the presence of a QPO in the middle NB (NB2) at the frequency $7.42 \pm 0.23 \mathrm{~Hz}$ (see the top-right panel of Figure 7). The F-test probability that the fit is improved by chance for an inclusion of this narrow feature is $\sim$ $3.1 \mathrm{e}-7$ and the significance of this $\mathrm{QPO}$ is $5.1 \sigma$. The quality factor of the NBO is found to be $\sim 4.88$ and $\mathrm{rms}$ is found to be $1.41 \pm 0.29 \%$. We also detect different types of band limited and red noise in the PDS. The types of the noise component detected in the different branches are listed in Table 6 and their best fit parameters along with rms are given in Table 7. In the top left HB (HB1), we find both LFN and HFN. In the HB2, we find only LFN. In the NB1, we detect only LFN. But in NB2 and NB3, we detect both VLFN and LFN. Again, in the FB1 we detect only LFN. In the FB2, we detect both LFN and VLFN. The FB3 and FB4 PDS show the presence of only VLFN feature. In Figure 7, we have shown three representative PDS at different sections of the Z-track. The integrated rms in the frequency range $0.07-100 \mathrm{~Hz}$ is found to be in the range of $1.3-4.75 \%$. 


\section{Discussion}

In this paper, we report the results obtained using $50 \mathrm{ks}$ LAXPC observations of the Z-source GX 17+2. In the HID, the source displayed all the three branches of the Z-diagram making it possible to study the broadband spectral evolution of the source in $3.0-80.0 \mathrm{keV}$ energy band along the complete Z-track.

Model 1 requires a strong hard X-ray tail (>30 keV) contributing $17 \%$ of the total $3.0-80.0 \mathrm{keV}$ flux in the upper HB (HB1). This hard X-ray tail can be described by a power-law model with photon index $\Gamma=2.95 \pm 0.02$. The hard X-ray tail becomes weaker as the source moves down the HB. In the HB2 the contribution of the hard tail is $\sim 6.1 \%$. As the source moves further down the NB the hard tail becomes much weaker contributing only $3-4 \%$ of the total flux. Model 2 has three components: emission from the thermal disk, blackbody emission from the boundary layer or NS surface and a power-law emission from a corona. This model is similar to that used by Lin et al. (2012). Note that Lin et al. (2012) used cutoff power-law (CPL) with cutoff at around $10 \mathrm{keV}$ to model the excess emission above $20 \mathrm{keV}$. However, we find a strong power-law emission extending above 30 $\mathrm{keV}$ without showing any signature of a cutoff. The power-law tail contributes $\sim 20 \%$ of the total flux in the upper HB when the spectra are fitted with Model 2. The powerlaw component diminishes as the source moves down the HB and further down in the NB. Though Model 2 predicts a significant power-law emission in the NB, its contribution above $40 \mathrm{keV}$ is almost negligible. This is due to fact that power-law component is steep in this region of the Z-track. The power-law again becomes stronger as the source moves up the FB. The effective inner disc radius $R_{\text {eff }}$ estimated using Model 2 increases as the source moves from the HB to the lower NB. The radius $R_{e f f}$ decreases as the source moves up the FB (see Figure 4b). A similar variation in the inner disc radius has been seen during the RXTE observations (Lin et al. 2012). The radius of neutron-star/boundary-layer obtained using Model 2 is only $\sim 4 \mathrm{~km}$. This radius has been obtained assuming isotropic emission from the neutron star surface. However, it is possible that the emission from the neutron star surface is anisotropic. The radius obtained assuming isotropic emission will be smaller compared to that obtained assuming anisotropic emission. We also corrected the radius for scattering in the the neutron star atmposphere assuming constant density atmosphere (Foster, Ross and Fabian 1986). In this case, the corrected radius is close to the radius of the neutron star $(8-10 \mathrm{~km})$.

It is worth mentioning that the contribution of the power-law component is found to be model dependent. However, both models (Model 1 and Model 2) predict the presence of a strong power-law component extending above $30 \mathrm{keV}$ in the FB.

Previous BeppoSAX observations of the source have also shown the presence of a strong 
hard X-ray tail in the upper HB which gradually faded as the source moved down the HB and completely vanished in the NB (Di Salvo et al. 2000). An another BeppoSAX observation of this source where the source displayed only HB (Farinelli et al. 2005) revealed a hard X-ray tail with photon index $\sim 2.8$ in the upper HB. The hard tail disappeared as the source moved to the lower HB (Farinelli et al. 2005). More importantly, we also find the evidence of strong hard X-ray tails in the middle and upper FB. The power-law tail is steep having photon index of $\sim 3.3-3.4$. The hard X-ray tail observed in this section of the FB contributes $\sim 15 \%$ of the total $3.0-80.0 \mathrm{keV}$ flux. Previously, hard X-ray tail has been detected in the FB of this source using HEXTE (High Energy X-ray Timing Experiment) data (Ding \& Huang 2015). Other sources which have shown hard tail in the FB are Sco X-1 and GX 5-1 (Asai et al. 1994; D'Amico et al. 2001). A hard X-ray tail with a flat or inverted index was reported in the FB of Sco X-1 (D'Amico et al. 2001; D'Ai et al. 2007) with HEXTE/RXTE. However, INTEGRAL observation of Sco X-1 revealed that at the top of the FB the hard X-ray tail disappeared (Revnivtsev et al. 2014; Di Salvo et al. 2006), suggesting transient nature of the hard X-ray tail.

GX 5-1 has also shown hard X-ray tails in the FB, however this component was fainter in the FB compared to the NB (Asai et al. 1994). In GX 349+2, the hard X-ray tail with photon index $\sim 1.9$ was found in the non-flaring state corresponding to the lower part of the NB (Di Salvo et al. 2001) and disappeared in the flaring branch. Similarly, 2001 BeppoSAX observations of GX 349+2 when the source was probably in the FB suggested the absence of the hard X-ray tail in the X-ray spectra of the source (Iaria et al. 2004).

Simultaneous X-ray and radio observations of GX $17+2$ indicate that the radio flux is correlated with the position on the HID (Penninx et al. 1998; Migliari et al. 2007). The radio flux decreases from the HB to the NB and is quenched in the FB (Migliari et al. 2007). The presence of the hard X-ray tail in the HB suggests that most probably Comptonization of the seed photons in a relativistic jet produces the hard X-ray tail (Di Salvo et al. 2000, 2002). However, present detection of a strong hard tail in the FB of GX 17+2, where radio flux disappears, can not be explained in this framework, a simultaneous radio/X-ray observation in this state would be needed to strengthen this statement. Other competing model is upscattering of the soft seed photons from the neutron star surface by relativistic electrons in bulk motion of converging flow (Titarchuk and Zannias 1998; Farinelli et al. 2009). However, in the FB radiation pressure can slow down or can stop the bulk flow causing quenching of hard X-ray tail (Farinelli et al. 2007). This suggests that a significant detection of hard $\mathrm{X}$-ray tail in the FB can not be explained in the frame work of bulk Comptonization. Hence, most probably the hard X-ray tail is produced by hybrid thermal/non-thermal electrons in the corona (Coppi 1999). Farinelli et al. (2005) used this model to explain the hard tail observed in the HB of GX 17+2. The hybrid thermal/non-thermal model was also applied 
to describe the hard excess seen in the spectra of Sco X-1 (D'Ai et al. 2007).

In Model 1 the emission from the source in the $3.0-80.0 \mathrm{keV}$ band is dominated by the contribution from an optically thick $(\tau \sim 7-10)$ and cool $(k T e \sim 3 \mathrm{keV})$ corona which is in accordance with the results obtained from the previous BeppoSAX observations (Di Salvo et al. 2000). The thermal Comptonized component contributes more than 80 per cent of the total flux. The spectral evolution of this source has been previously studied using BeppoSAX data (Di Salvo et al. 2000). However, BeppoSAX data revealed only the HB and NB of the source. During our observations, source has shown extended FB making it possible to study the spectral behaviour of the source along a complete Z-track.

As the source GX 17+2 moves along the HB and then down the NB, the optical depth $\tau$ of the corona decreases which is in accordance with the previously observed behaviour exhibited by the source GX 17+2 (Di Salvo et al. 2000). The increase in the optical depth along the FB is similar to that observed in the Z-source LMC X-2 previously (Agrawal and Misra 2009). The decrease in optical depth along the NB and then increase along the FB has also been previously reported in the Z-source GX 349+2 (Agrawal and Sreekumar 2003). The electron temperature $k T_{e}$ in the $\mathrm{HB}$ and NB is $\sim 3.2 \mathrm{keV}$. Similar values of $k T_{e}$ was found during BeppoSAX observation of the source in the HB and NB (Di Salvo et al. 2000). In the $\mathrm{FB}$, the $k T_{e}$ is slightly lower.

The evolution of the Comptonized spectrum can be explained in terms of increasing accretion rate scenario. In this scenario, as the soft photon supply from neutron star surface/disc increases, the part of the material from the corona interacts with these photons and settles down in the disc. This causes the reduction in the optical depth of the corona. This explains the observed behaviour of the source along the HB to NB. Similarly, as the accretion rate further increases radiation pressure pushes a part of the material from the accretion disc into the corona causing it to become optically thick (see also Agrawal and Sreekumar 2003). Comptonization parameter $y$, which is a measure of relative energy gain, decreases along the HB and then upto lower part of the NB (from NB1 to NB3) causing spectrum to become softer. Similarly increase in the $y$-parameter causes the source to move up on the FB. The reported variation in the $y$ parameter is in accordance with that observed in the source GX 349+2 (Agrawal and Sreekumar 2003) and LMC X-2 (Agrawal and Misra 2009).

Model 3 which includes a reflection component from the ionized disc provides equally good fit to the spectra of the source. The reflection component does not require a relativistic smearing, suggesting that iron line is narrow. This is supported by the fact that inner rim of the disk is truncated away from the compact object $\left(10-45 R_{g}\right)$. The reflection fraction varies between $0.3-0.5$ and attains the highest values near the NB-FB vertex (see Table 4). We also note that the equivalent width of the iron line derived using Model $\mathbf{1}$ is correlated with 
the reflection fraction of Model 3 which further supports that iron line is due to reflection of Comptonized emission from the truncated disc. Model 4, which includes the reflecction of the boundary layer emission from the ionized accretion disk, is also a good description of the spectra. Here, we have also used a reletivistic convolution which suggests that the accretion disc is very far from the compact object $\left(10-20 R_{I S C O}\right)$. This suggests that the space between the compact object and inner rim of accretion disc should be filled with some hot plasma. Hence, Model 3 which invokes reflection of the Comptonized emission emitted by this plasma by a ionized accretion disc seems to be a more promising scenario. Also reflection fraction (from Model 3) is much better correlated (correlation coeffficient $=0.94$ ) with equivalent width of iron line compared to reflection fraction obtained using BBREFL model (correlation coefficient $=0.63$ ), again suggesting that Model $\mathbf{3}$ is a better description.

Modelling of the relativistic iron line with diskline and reflection models suggests that the accretion disc is truncated away from the neutron star. However, modelling of the reflection signature observed with the NuSTAR data suggests that the accretion disc in this source lies

close to the inner most stable circular orbit (ISCO) (Ludham et al. 2017), although these authors do not mention the spectral branch where they have detected the reflection feature.

A normal branch oscillation (NBO) with frequency $7.42 \pm 0.29 \mathrm{~Hz}$ is detected at the NB2 section of the NB. If we consider Model 1 as correct description of the spectra, the thermal Comptonized emission is the only dominant component in the $3.0-80.0 \mathrm{keV}$ energy band. It is more probable that an optically thick central corona is producing this emission component by up-scattering of the soft seed photons supplied by the neutron star surface. Hence, it seems that the radiation feedback mechanism with this hot central corona is probably producing the NBO (see Fortner, Lamb \& Miller 1989) observed in this source.

\section{Acknowledgements}

We thank anonymous reviewer for providing useful suggestions which improved the quality of the paper. This research has made use of the data obtained through GT phase of AstroSat observation. Authors thank GD, SAG; DD, PDMSA and Director, URSC for encouragement and continuous support to carry out this research. This work has used the data from the LAXPC Instruments developed at TIFR, Mumbai and the LAXPC POC at TIFR is thanked for verifying and releasing the data via the ISSDC data archive. We thank the AstroSat Science Support Cell hosted by IUCAA and TIFR for providing the LaxpcSoft software which we used for LAXPC data analysis. 


\section{REFERENCES}

Agrawal P.C., Yadav J.S., Antia H.M., Dedhia Dhiraj, Chauhan Jai Verdhan, Manchanda R.K., Chitnis V.R. et al., 2017, JApA, 38,30

Agrawal V.K., Nandi Anuj, Girish V., Ramadevi M.C., 2018, MNRAS, 477, 5437

Agrawal V.K., Sreekumar P., 2003, MNRAS, 346, 933

Agrawal V.K., Misra R., 2009, MNRAS, 398, 1352

Antia H.M., Yadav J.S, Agrawal P.C, Verdhan Chauhan Jai, Manchanda R.K.,Chitnis Varsha, Paul B., Dedhia Dhiraj et al.,2017, ApJS, 231,10

Asai K., Dotani T., Mitsuda K., Nagase F., Kamado Y., Kuulkers E., Breedon L.M., 1994, PASJ, 46, 479

Ballantyne D. R. 2004, MNRAS, 351, 57

Barret D., 2001, AdSpR, 28, 307

Cackett E.M., Miller J. M., Ballantyne D.R., Barret D., Bhattacharyya S., 2010, ApJ, 720, 205

Coppi P.S., 1999, High Energy Process in Accreting Blackholes, ASP Conference Series, 161, Juri Poutanen and Ronal Svensson eds.

D’Ai A., Zycki P., Di Salvo T., Iaria R., Lavagetto G., Robba N.R., 2007, ApJ, 667, 411

D’Amico F., Heindl W.A., Rothschild R.E., Gruber D.E., 2001, ApJ, 547, L147

Dauser T., Wilms J., Reynolds C. S., Brenneman L. W. 2010, MNRAS,409, 1534

Davis S.W., Blaes O.M., Hubeny I., Turner N.J., 2005, 621, 372

Ding G.Q., Huang C.P., 2015, JApA, 36, 335

Ding G.Q., Zhang S.N., Wang N., Qu J.L., Yan S.P., 2011, AJ, 142, 34

Done C., Zycki P.T. and Smith D.A, 2002, MNRAS, 331, 453

Di Salvo T., Goldoni P., Stella L., van der Klis M., Bazzano A., Burderi L., Farinelli R., Frontera F. et al, 2006, ApJL, 649, 91

Di Salvo T., Stella L., Robba N.R., van der Klis M., Burderi L., Israel G.L, Homan J., Compana S. et al., 2000, ApJ, 544, L119 
Di Salvo T. et al., 2002, A\&A, 386, 535

Di Salvo T., et al., 2001,ApJ, 554, 49

Fabian A.C., Rees M. J., Stella L., \& White N.E., 1989, MNRAS, 238, 729

Farinelli R., Paizis A., Landi R., and Titarchuk L.,2009, A\&A, 498, 509

Farinelli R., Frontera F., Zdziarski A.A., Stella L., Zhang S.N., van der Klis M., Masetti N., Amati L., 2005, A\&A, 434, 25

Farinelli R., Titarchuk L., and Frontera F., 2007, ApJ, 662, 1167

Fortner B., Lamb F.K., \& Miller G. S., 1989, Nature, 342, 775

Foster A.J., Ross R.R., Fabian A. C., 1986, MNRAS, 221, 409

Hasinger G., van der Klis M., 1989, A\&A, 225, 79

Homan J., van der Klis M., Wijnands R., Belloni,T., Fender R., Klein-Wolt, M., Casella P., Mendez M., et al., 2007, ApJ, 656, 420

Homan J., van der Klis M., Jonker P.G., Wijnands R.,Kuulkers E., Mendez M., Lewin W.H.G., 2002, ApJ, 568, 878

in't Zand J.J.M., Verbunt F., Strohmayer T.E., Bazzano A., Cocchi M., Heise J., van Kerkwijk M.H., Muller J.M. et al., 1999, A\&A, 345, 100

Iaria R., Burderi L., Di Salvo T., La Barbera A., Robba N.R., 2001, ApJ, 547, 412

Iaria R., Di Salvo T., Robba N.R., Burderi L., Stella L., Frontera F., van der Klis M.,2004, ApJ, 600,358

Iaria R., Lavagetto G., Di Salvo T., D’Ai A., Burderi L., Stella L., Robba N.R., 2006, Chin. J. Astron. Astrophys., 6, 257

Jonker P.G., Wijnands R., van der Klis M., Psaltis D, Kuulkers E., Lamb F.K., 1998, ApJL, 499, 191

Jonker, P.G., van der Klis M., Wijnands R., Homan J., van Paradijs J., Mendez M., Ford E.C., Kuulkeres E., Lamb F.K., ApJ, 2000, 537, 374

Jonker P.G., van der Klis M., Homan J., Mendez M., Lewin W.H.G., Wijnands R., Zhang W., 2002, MNRAS, 333, 665 
Kolehmainen M., Done C., Trigo M.D., 2011, MNRAS, 416, 311

Kuulkers E., den Hartog P.R., in’t Zand J.J.M., et al. 2003, A\&A, 399, 663

Lin D., Remillard R.A., Homan J., Barret D., 2012, ApJ, 756, 34

Lin D., Remillard R.A. and Homan J., 2009, ApJ, 696, 1257

Ludham R.M., Miller J.M., Bachetti M., Barret D., Bostrom A.C., Cackett E.M., Degenaar N., Disalvo T., et al., 2017, ApJ, 836, 140

Migliari S., Miller-Jones J. C. A., Fender R. P., Homan J., Di Salvo T., Rothschild R. E., Rupan M. P., Tomsick J. A., 2007, ApJ, 671, 706

Mitsuda K., et al., 1984, PASJ, 36, 741

Penninx W., Lewine W. H. G., Zijlstra A. A., Mitsuda K., van Paradijs J., 1988, Natur, 336, 146

Remillard R.A.,\& Lin D., The ASM Team at MIT and NASA/GSFC 2006, Astron. Tel. 696

Revnivtsev M.G., Tsygankov S.S., Churazov E.M., Krivonos R.A.,2014, MNRAS, 445, 1205

Sanna A., Mendez M., Altamirano D., Homan J., Casella P., Belloni T., Lin D., Van der Klis M., et al., 2010, MNRAS, 408, 622

Shimura T., Takahara F., 1995, ApJ, 445, 780

Sleator C.C., Tomsick J.A., King A.L., Miller J.M., Boggs S.E., Bachetti M., Barret D., Chenevez J., 2016, ApJ, 827, 134

Titarchuk L. \& Zannias T., 1998, ApJ, 493, 863

van der Klis M., 1995, in X-ray binaries,ed. W.H.G. Lewin, J. van Paradijs and E.P.J. van den Heuvel (Cambridge Astrophysics Ser 26, Cambridge:Cambridge Univ. Press), 252

van der Klis M., Swank J.H., Zhang W., Jahoda K., Morgan E.H., Lewin W.H.G., Vaughan B. and van Paradijs J., 1996, ApJL, 469, 1

Wijnands R., Homan J., van der Klis M., Kuulkers E., van Paradijs J., Lewin W.H.G., Lamb F.K., Psaltis D., 1998a, ApJL, 493,87

Wijnands R., Mendez M., van der Klis M., Psaltis D., Kuulkers E., and Lamb F.K., 1998b, ApJL, 504,35 
Wijnands R., Homan J., van der Klis M., Mendez M., Kuulkers E., van Paradijs J., Lewin W.H.G., Lamb F.K. et al.,1997, ApJL, 490, 157

Yadav J.S.,Agrawal P.C.,Antia H.M.,Chauhan Jai Verdhan,Dedhia Dhiraj, Katoch T., Madhwani P., Manchanda R.K.,et al., 2016, SPIE, 9905, 1

Zhang W., Strohmayer T.E. and Swank J.H.,1998, ApJL, 500, 167

Zdziarski A. A., Johnson W.N., Magdziarz P., 1996, MNRAS, 283, 193

Zhang W., Jahoda K., Swank J.H., Morgan E.H. and Giles A.B., 1995, ApJ, 449, 930 